Pacific Journal of Mathematics

THE EVALUATION MAP AND EMP SEQUENCES 


\title{
THE EVALUATION MAP AND EHP SEQUENCES
}

\author{
GEORGE E. LANG, JR.
}

Let $L(\Sigma B, X)$ be the space of maps from $\Sigma B$ (the reduced suspension of $B$ ) to $X$ with the compact-open topology, let $\measuredangle: \Sigma B \rightarrow X$ and $L(\Sigma B, X ; \measuredangle)$ the path component of $L(\Sigma B, X)$ containing $\ell$. For nice spaces the evaluation map $\omega$ : $L(\Sigma B, X, \ell) \rightarrow X$ defined by $\omega(f)=f\left(^{*}\right)$ is a fibration and gives rise to a long exact sequence in homotopy. The purpose of this paper is to show that the boundary map in that long exact sequence can be given by a generalized Whitehead product and that the sequence generalizes the $E H P$ sequence of $\mathrm{G}$. W. Whitehead.

1. Preliminary definitions. All spaces are assumed to be $C W$ complexes with base point at a vertex. Maps are base point preserving. The cartesian product $A \times B$ is assumed to be based at $\left(a_{0}, b_{0}\right)$, the unit inverval, $I$, is based at 0 , and quotient spaces are based at the image of the base point under the natural quotient map. Where the space is clear * will denote the base point as well as the constant map with image at the base point.

We use the following notations. $L(A, B)$ will denote the space of maps from $A$ to $B$ with the compact-open topology and $L(A, B ; \ell)$ the path component of $L(A, B)$ containing $\ell: A \rightarrow B . \quad L_{0}(A, B)$ and $L_{0}(A, B ; \ell)$ will denote the space of base point preserving maps in $L(A, B)$ and $L(A, B ; \ell)$ respectively. Let $A \vee B$ and $A \# B$ denote the one point union and smash product respectively.

Since spaces are assumed to be $C W$ complexes the smash product can be taken as $A \times B$ with $A \vee B$ identified with $\left(a_{0}, b_{0}\right) . \quad q: A \times B \rightarrow$ $A \# B$ will denote the quotient map. Note that $S^{p+q}=\Sigma^{p} S^{q}=S^{p} \# S^{q}$, $\Sigma^{p} A=S^{p} \# A$, and $\Sigma(A \vee B)=\Sigma A \vee \Sigma B$.

Let $p_{1}, p_{2}: A \times B \rightarrow A \vee B$ be defined by $p_{1}(a, b)=a \vee b_{0}$ and $p_{2}(a, b)=a_{0} \vee b$. Define $k: \Sigma(A \times B) \rightarrow \Sigma A \vee \Sigma B$ by $k=\Sigma p_{1}+\Sigma p_{2}$ $\Sigma p_{1}-\Sigma p_{2}$. Since $k \mid \Sigma(A \vee B)$ homotopically trivial, by the homotopy extension property there is a map $k^{\prime}: \Sigma(A \times B) \rightarrow \Sigma A \vee \Sigma B$, homotopic to $k$, such that $k^{\prime} \mid \Sigma(A \vee B)={ }^{*}$. $k^{\prime}$ then induces a map $\tilde{k}: \Sigma(A \# B) \rightarrow$

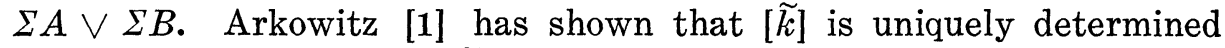
by the requirement $k \cong \tilde{k} \circ \Sigma q$. The following definition is due to Arkowitz [1].

Definition 1.1. For $\alpha=[f] \in[\Sigma A, X]$ and $\beta=[g] \in[\Sigma B, X]$, the generalized Whiteheal product $[\alpha, \beta]$ is defined by $[\alpha, \beta]=[(f \vee g) \circ \widetilde{k}]$ $\in[\Sigma(A \# B) X]$. 
Hardie shows (Theorem 2.3 in [2]) that the map $\Sigma p_{1}+\Sigma p_{2}+$ $\Sigma q: \Sigma(A \times B) \rightarrow \Sigma A \vee \Sigma B \vee \Sigma(A \# B)$ is a homotopy equivalence for $A$ and $B C W$ complexes with a single vertex. Then there is a map $\phi: \Sigma(A \# B) \rightarrow \Sigma(A \times B)$ such that $\Sigma q \circ \phi \cong 1_{\Sigma(A * B)}$.

Definition 1.2. If $f: A \times B \rightarrow X$, where $A$ and $B$ have a single vertex, the element obtained from $f$ by the generalized Hopf construction is defined to be the map $\Sigma f \circ \phi: \Sigma(A \# B) \rightarrow \Sigma X$.

Hardie shows in [2] that if $A$ and $B$ are spheres, Definition 1.2 reduces to the classical definition of the Hopf construction.

Let $\phi_{r}: S^{r} \rightarrow S^{r} \vee S^{r}$ be the map which identifies the equator of $S^{r}$. G. W. Whitehead (Theorem 1.17 in [6]) shows for $n<p+q+$ $\min (p, q)-3$ that $\pi_{n}\left(S^{p} \vee S^{q}\right)=\pi_{n}\left(S^{p}\right) \oplus \pi_{n}\left(S^{q}\right) \oplus \pi_{n}\left(S^{p+q-1}\right)$. Let $Q: \pi_{n}\left(S^{p} \vee S^{q}\right) \rightarrow \pi_{n}\left(S^{p+q-1}\right)$ be the natural projection onto the direct summand $\pi_{n}\left(S^{p+q-1}\right)$.

DefinItION 1.3. For $n<3 r-3$ the generalized Hopf invariant $\widetilde{H}: \pi_{n}\left(S^{r}\right) \rightarrow \pi_{n}\left(S^{2 r-1}\right)$ is defined by $\widetilde{H}=Q \circ \phi_{r^{*}}$.

Definition 1.4. For $\lambda=[\ell] \in[\Sigma B, X]$ the $\lambda$-Whitehead homomorphism $P_{\lambda}:[\Sigma A, X] \rightarrow[\Sigma(A \# B), X]$ is defined by $P_{\lambda}(\alpha)=[\alpha, \lambda]$.

Definition 1.5. If $F: A \rightarrow L(B, X)$ the map $G: A \times B \rightarrow X$ given by $G(a, b)=F(a)(b)$ is said to be an associated map for $F$.

2. The $\lambda$-component $E H P$ sequence. The purpose of this section is to show that the map $P_{2}$ of Definition 1.4 is embedded in a long exact sequence resulting from the fibration $\omega: L(\Sigma B, X ; \measuredangle) \rightarrow X$. Each $\lambda \in[\Sigma B, X]$ determines a path component of $L(\Sigma B, X)$ and $\omega$ restricted to each path component determine a fibration and a long exact sequence. In $\S 3$ the relationship between these sequences and the James suspension sequence is explored and it is shown that G. W. Whitehead's EHP sequence [7] is a special case of an $\iota_{n}$-component EHP sequence where $\iota_{n}=\left[1_{S^{n}}\right]$ in $\pi_{n}\left(S^{n}\right)$.

Lemma 2.1. For $\ell \in L_{0}(\Sigma B, X), L_{0}(\Sigma B, X$; *) is homotopy equivalent to $L_{0}(\Sigma B, X ; \ell)$.

Proof. Let $\hat{\ell}: L_{0}\left(\Sigma B, X ;{ }^{*}\right) \rightarrow L_{0}(\Sigma B, X ; \ell)$ be defined by $\hat{\ell}(g)=$ $g+\ell$ and $\hat{\ell}^{-1}: L_{0}(\Sigma B, X ; \ell) \rightarrow L_{0}\left(\Sigma B, X ;{ }^{*}\right)$ by $\hat{\ell}^{-1}(g)=g-\ell$. Then it is clear that $\hat{\ell}^{-1}$ is a two sided homotopy inverse of $\hat{\ell}$.

In remaining parts of this section the map $\hat{\ell}$ will be taken to be given by 


$$
\hat{\ell}(g)(b, t)= \begin{cases}g\left(b, \frac{5}{4} t\right) & 0 \leqq t \leqq \frac{4}{5} \\ \iota(b, 5 t-4) & \frac{4}{5} \leqq t \leqq 1\end{cases}
$$

Lemma 2.2. $[\Sigma(A \# B), X]$ is isomorphic to $\left[A, L_{0}\left(\Sigma B, X ;{ }^{*}\right)\right]$.

This fact is well know. For the remainder of this section the isomorphism will be denoted by $\theta:[\Sigma(A \# B), X] \rightarrow\left[A, L_{0}\left(\Sigma B, X ;{ }^{*}\right)\right]$ defined as follows. If $f: \Sigma(A \# B) \rightarrow X, \theta(f)(a)$ is the map taking $(b, t)$ to $f((a, b), t)$ in $X$.

Definition 2.3. $A @ B$ is defined as $A \times B$ with $A \times\left\{b_{0}\right\}$ identified with $\left(a_{0}, b_{0}\right)$.

Let $m: A \times \Sigma B \rightarrow(A \# \Sigma B) \vee(A @ \Sigma B)$ be defined by

$$
\left(m(a,(b,))= \begin{cases}\left(a,\left(b, \frac{5}{4} t\right)\right) \vee * & 0 \leqq t \leqq \frac{4}{5} \\ * \vee(a,(b, 5 t-4)) & \frac{4}{5} \leqq t \leqq 1\end{cases}\right.
$$

Now let $G: A \# \Sigma B \rightarrow X$ be a map associated with $[g] \in$ $\left[A, L_{0}\left(\Sigma B, X ;{ }^{*}\right)\right], \iota \in L_{0}(\Sigma B, X)$, and $p_{2}: A @ \Sigma B \rightarrow \Sigma B$ the natural projection.

The following lemma can be easily verified.

LEMMA 2.4. $\left(G \vee\left(\ell \circ p_{2}\right)\right) \circ m: A \times \Sigma B \rightarrow X$ is an associated map for $\hat{\iota}_{*}([g]) \in\left[A, L_{0}(\Sigma B, X ; \iota)\right]$.

Let $h_{1}: A \times S B \rightarrow \Sigma(A \times B)$ be defined by $h_{1}(a,(b, t))=((a, b), t)$, where $S A$ is the unreduced suspension.

By the homotopy extension property the quotient map $q_{1}: S B \rightarrow \Sigma B$ is a homotopy equivalence. Its homotopy inverse will be denoted $q_{1}^{-1}: \Sigma B \rightarrow S B$.

LEMma 2.5. Let $\alpha=[f] \in[\Sigma A, X]$ and $\lambda=[\ell] \in[\Sigma B, X]$, then $(f \vee \ell) \circ\left(\Sigma p_{1}+\Sigma p_{2}-\Sigma p_{1}-\Sigma p_{2}+\Sigma p_{2}\right) \circ h_{1} \circ\left(1_{A} \times q_{1}^{-1}\right): A \times \Sigma B \rightarrow X$ is a map associated with $\hat{\ell}_{*} \circ \theta([\alpha, \lambda]) \in\left[A, L_{0}(\Sigma B, X ; \ell)\right]$.

Proof. Let $m_{1}: \Sigma(A \times B) \rightarrow \Sigma(A \times B) \vee \Sigma(A \times B)$ be given by

$$
m_{1}((a, b), t)= \begin{cases}\left((a, b), \frac{5}{4} t\right) \vee * & 0 \leqq t \leqq \frac{4}{5} \\ * \vee((a, b), 5 t-4) & \frac{4}{5} \leqq t \leqq 1 .\end{cases}
$$


Consider the following diagram:

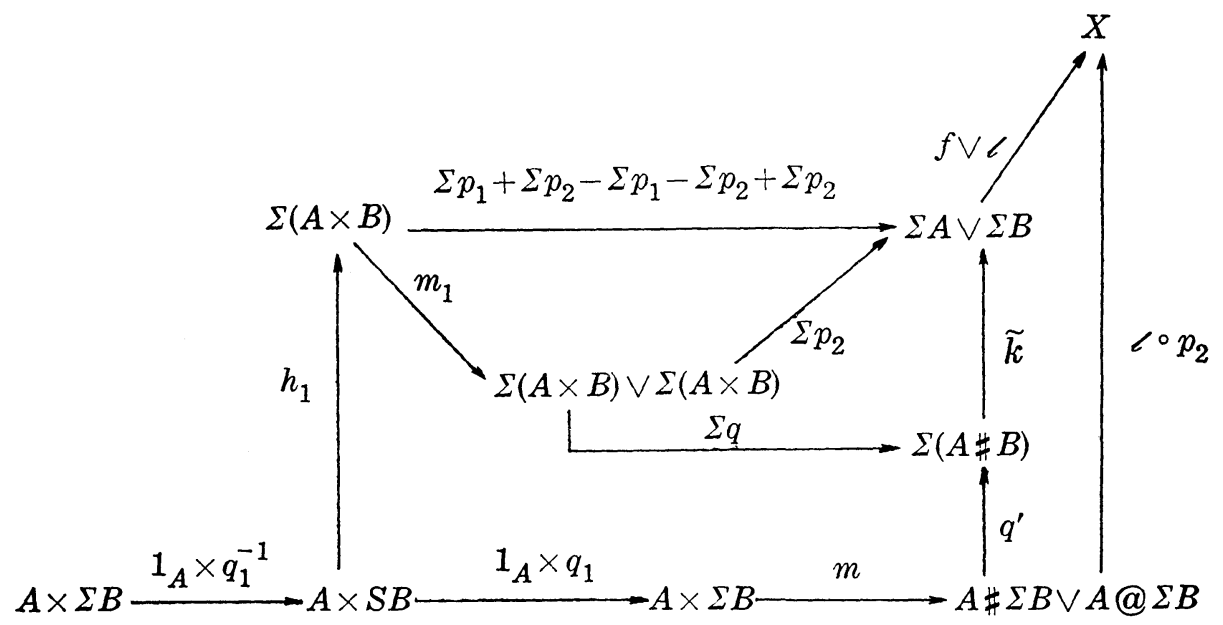

$q^{\prime}: A \# \Sigma B \rightarrow \Sigma(A \# B)$ is the homomorphism defined by $q^{\prime}(a,(b, t))=$ $((a, b), t)$ and $k$ is as in Definition 1.1. It is easiest to check the homotopy commutativity of this diagram by looking first at the lower four fifths of the $t$ coordinate in $S B$ and then at the upper fifth.

$$
\text { Part 1. } \Sigma p_{1}+\Sigma p_{2}-\Sigma p_{1}-\Sigma p_{2}+\Sigma p_{2} \cong\left((\tilde{k} \circ \Sigma q) \vee \Sigma p_{2}\right) \circ m_{1} \text {. }
$$

The lower four fifths of $\Sigma(A \times B)$ is mapped in one case by $\Sigma p_{1}+\Sigma p_{2}-\Sigma p_{1}-\Sigma p_{2}$ and in the other by $\tilde{k} \circ \Sigma q$. But these are homotopic by the definition of $\tilde{k}$. The upper fifth is mapped by $\Sigma p_{2}$ in either case.

Part 2. $(f \vee \ell) \circ\left(\tilde{k} \circ \Sigma q \vee \Sigma p_{2}\right) \circ m_{1} \circ h_{1}=\left(\left((f \vee \ell) \circ \tilde{k} \circ q^{\prime}\right) \vee \ell \circ p_{2}\right) \circ m \circ$ $\left(1_{A} \times q_{1}\right)$.

On the lower four fifths the maps "meet" at $\Sigma(A \# B)$. In either case the point $(a,(b, t)) \in A \times S B$ is mapped to $((a, b),(5 / 4) t) \in \Sigma(A \# B)$. On the upper fifth both maps are given by taking $(a,(b, t))$ to $\iota((b, 5 t-4)$ in $X$.

By Lemma 2.4 and the definition of $[\alpha, \lambda],\left(\left((f \vee \ell) \circ \tilde{k} \circ q^{\prime}\right) \vee\left(\ell \circ p_{2}\right)\right) \circ$ $m$ is an associated map for $\iota_{*} \theta([\alpha, \lambda])$. This is the lower route in the above diagram. Since $q_{1}$ and $q_{1}^{-1}$ are homotopy inverses

$$
\begin{aligned}
(((f & \left.\left.\vee \ell) \circ \tilde{k} \circ q^{\prime}\right) \vee \ell \circ p_{2}\right) \circ m \\
& \cong\left(\left((f \vee \ell) \circ \tilde{k} \circ q^{\prime}\right) \vee \ell \circ p_{2}\right) \circ m \circ\left(1_{A} \times q_{1}\right) \circ\left(1_{A} \times q_{1}^{-1}\right) \\
& \cong(f \vee \ell) \circ\left(\tilde{k} \circ \Sigma q \vee \Sigma p_{2}\right) \circ m_{1} \circ h_{1} \circ\left(1_{A} \times q_{1}^{-1}\right) \\
& \cong(f \vee \ell) \circ\left(\Sigma p_{1}+\Sigma p_{2}-\Sigma p_{1}-\Sigma p_{2}+\Sigma p_{2}\right) \circ h_{1} \circ\left(1_{A} \times q_{1}^{-1}\right) .
\end{aligned}
$$

The last two homotopies follow from Part 2 and Part 1 respectively. But the last map is the one claimed to be an associated map for 
$\hat{\iota}_{*} \theta([\alpha, \lambda])$ and the lemma is proven.

For $\lambda=[\ell] \in[\Sigma B, X]$ the evaluation map $\omega: L(\Sigma B, X ; \ell) \rightarrow X$ is a fibration with fiber $L_{0}(\Sigma B, X ; \ell)$. Then there is a long exact sequence of homotopy groups

$$
\begin{aligned}
& \cdots \longrightarrow\left[\Sigma^{r+1} A, X\right] \stackrel{\partial}{\longrightarrow}\left[\Sigma^{r} A, L_{0}(\Sigma B, X ; \ell)\right] \stackrel{i_{*}}{\longrightarrow} \\
& {\left[\Sigma^{r} A, L(\Sigma B, X ; \iota)\right] \stackrel{\omega_{*}}{\longrightarrow}\left[\Sigma^{r} A, X\right] \stackrel{\partial}{\longrightarrow} \cdots \longrightarrow[\Sigma A, X] \stackrel{\partial}{\longrightarrow}} \\
& {\left[A, L_{0}(\Sigma B, X ; \iota)\right] \stackrel{i_{*}}{\longrightarrow}[A, L(\Sigma B, X ; \iota)] \stackrel{\omega_{*}}{\longrightarrow}[A, X],}
\end{aligned}
$$

where exactness at the last two stages is as pointed sets. Recall that Lemma 2.2 shows there is an isomorphism $\theta:[\Sigma(A \# B), X] \rightarrow$ $\left[A, L\left(\Sigma B, X ;{ }^{*}\right)\right]$.

THEOREM 2.6. For $\alpha \in\left[\Sigma^{r} A, X\right], \partial(\alpha)=\hat{\ell}_{*} \circ \circ \circ P_{\lambda}(\alpha)$.

Proof. Let $\alpha$ be represented by a map $f: \Sigma^{r} A \rightarrow X$ and let $q_{2}: C\left(\Sigma^{r-1} A\right) \rightarrow \Sigma^{r} A$ be the natural quotient map from the cone to the suspension. Define $F: C\left(\Sigma^{r-1} A\right) \times S B \rightarrow \Sigma\left(\Sigma^{r-1} A\right) \vee \Sigma B$ by

$$
F((a, r),(b, t))= \begin{cases}q_{2}(a, r+3 t) \vee * & 0 \leqq t \leqq \frac{1}{3} r \leqq-3 t+1 \\ * & 0 \leqq t \leqq \frac{1}{3} r \geqq-3 t+1 \\ * \vee q_{1}(b, 3 t-1) & \frac{1}{3} \leqq t \leqq \frac{2}{3} \\ * & \frac{2}{3} \leqq t \leqq 1 r \geqq 3 t-2 \\ q_{2}(a, 3+r-3 t) \vee * & \frac{2}{3} \leqq t \leqq 1 r \leqq 3 t-2\end{cases}
$$

where $(a, r) \in C\left(\Sigma^{r-1} A\right), r$ being the level on the cone and $(b, t) \in S B$, $t$ being the level on the suspension. At $t=1 / 3$ or $2 / 3$ and on the lines $r=-3 t+1$ and $r=3 t-2$, the image of $F$ is at ${ }^{*}$ and $F$ is well defined and continuous at these points. Since $F$ is independent of $a$ at $r=1$ and independent of $b$ at $t=1$ and $t=0, F$ is well defined. Let $\Sigma^{r-1} A \times S B \rightarrow C\left(\Sigma^{r-1} A\right) \times S B$ be induced by including $\Sigma^{r-1} A$ at the 0 level of $C\left(\Sigma^{r-1} A\right)$. Consider the following diagram:

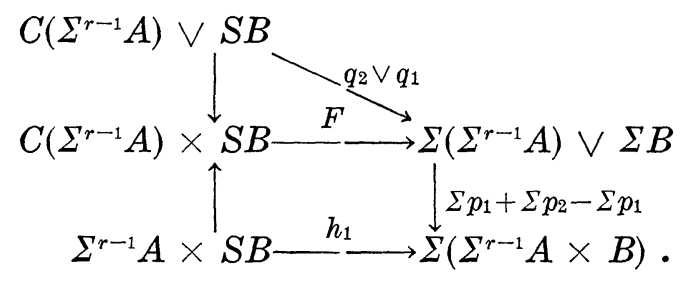


The map $\left(\Sigma p_{1}+\Sigma p_{2}-\Sigma p_{1}\right) \circ h_{1}$ is given by

$$
(a,(b, t)) \longrightarrow \begin{cases}(a, 3 t) \vee * & 0 \leqq t \leqq \frac{1}{3} \\ * \vee q_{1}(b, 3 t-1) & \frac{1}{3} \leqq t \leqq \frac{2}{3} \\ (a, 3-3 t) \vee * & \frac{2}{3} \leqq t \leqq 1\end{cases}
$$

But this is the same as $F((a, 0),(b, t))$, that is $F \mid \Sigma^{r-1} A \times S B$. Therefore the lower square commutes. In the upper triangle, when $t=0$ (the base point of $S B), F((a, r),(b, 0))=q_{2}(a, r)$ by definition. At the base point of $C\left(\Sigma^{r-1} A\right)$ consider

$$
F\left(\left(a_{0}, 0\right),(b, t)\right)= \begin{cases}q_{2}\left(a_{0}, 3 t\right) \vee *{ }^{*} & 0 \leqq t \leqq \frac{1}{3} \\ * \vee q_{1}(b, 3 t-1) & \frac{1}{3} \leqq t \leqq \frac{2}{3} \\ q_{2}\left(a_{0}, 3-3 t\right) \vee *=^{*} & \frac{2}{3} \leqq t \leqq 1 .\end{cases}
$$

But this is clearly homotopic to ${ }^{*} \vee q_{1}$, thus the upper triangle commutes up to homotopy. Now consider the map $\widetilde{F}: C\left(\Sigma^{r-1} A\right) \times \Sigma B \rightarrow$ $X$ given by $\widetilde{F}=(f \vee \ell) \circ F \circ\left(1_{C\left(\Sigma^{r}-1_{A}\right)} \times q_{1}^{-1}\right) . \quad \widetilde{F}$ is then an associated map for an element of $\left[\left(C\left(\Sigma^{r-1} A\right), \Sigma^{r-1} A\right),\left(L(\Sigma B, X ; \ell), L_{0}(\Sigma B, X ; \ell)\right)\right]$. Since $\widetilde{F} \mid C\left(\Sigma^{r-1} A\right) \times *$ is given by $f \circ q_{2}, \widetilde{F}$ is associated to the class $[f] \in\left[\Sigma^{r} A, X\right]$ under the bijection (see p. 104 in [5]) $\omega_{*}:\left[\left(C\left(\Sigma^{r-1} A\right)\right.\right.$, $\left.\left.\Sigma^{r-1} A\right),\left(L(\Sigma B, X ; \ell), L_{0}(\Sigma B, X ; \ell)\right)\right] \rightarrow\left[\Sigma^{r} A, X\right]$. Then by definition of the boundary homomorphism, $\partial([f])=\partial(\alpha)$ has associated map $\widetilde{F} \mid \Sigma^{r-1} A \times \Sigma B$. But by commutativity of the above diagram $\widetilde{F} \mid \Sigma^{r-1} A \times$ $\Sigma B \cong(f \vee \ell) \circ\left(\Sigma p_{1}+\Sigma p_{2}-\Sigma p_{1}\right) \circ h_{1} \circ\left(1_{\Sigma^{r-1}} A \times q_{1}^{-1}\right)$ and by Lemma 2.5 this is an associated map for $\hat{\ell}_{*} \circ \theta \circ([\alpha, \lambda])=\hat{\ell}_{*} \circ \theta \circ P_{\lambda}(\alpha)$.

The existence of the $\lambda$-component $E H P$ sequence now can be shown. Let $i_{*}^{\prime}:\left[\Sigma\left(\Sigma^{r-1} A \# B\right), X\right] \rightarrow\left[\Sigma^{r-1} A, L(\Sigma B, X ; \ell)\right]$ be given by $i_{*}^{\prime}=i_{*} \circ \hat{\ell}_{*} \circ \theta$.

THEOREM 2.7. There is a long exact sequence

$$
\begin{aligned}
& \cdots \longrightarrow\left[\Sigma^{r} A, L(\Sigma B, X ; \ell)\right] \stackrel{\omega_{*}}{\longrightarrow}\left[\Sigma^{r} A, X\right] \stackrel{P_{\lambda}}{\longrightarrow} \\
& {\left[\Sigma\left(\Sigma^{r-1} A \# B\right) ; X\right] \stackrel{i_{*}^{\prime}}{\longrightarrow}\left[\Sigma^{r-1} A, L(\Sigma B, X ; \iota)\right] \longrightarrow \cdots}
\end{aligned}
$$

Proof. Since $\hat{\ell}_{*}$ and $\theta$ are isomorphisms, the exactness of this sequence is immediate from the exactness of the homotopy exact sequence of the fibration $\omega_{*}: L(\Sigma B, X ; \measuredangle) \rightarrow X$ and Theorem 2.6. 
3. The Whitehead and James sequences. The purpose of this section is to compare the $\lambda$-component $E H P$ sequence with the classical $E H P$ sequence of George W. Whitehead [7] and the suspension sequence of I. M. James [4]. The spaces $A$ and $B$ will be assumed to be $C W$ complexes with a single vertex. For $\alpha \in[A, L(\Sigma B, X ; \ell)]$ the element $H(\alpha) \in[\Sigma(A \# \Sigma B), \Sigma X]$ is defined by the element obtained from a map associated with $\alpha$ by the Hopf construction of Definition 1.2. The homomorphism $E:[A \# \Sigma B, X] \rightarrow[\Sigma(A \# \Sigma B), \Sigma X]$ is defined by $E([f])=$ $[\Sigma f]$.

LEMMA 3.1. The following diagram commutes:

$$
\begin{gathered}
{[A \# \Sigma B, X] \stackrel{E}{\longrightarrow}[\Sigma(A \# \Sigma B), \Sigma X]} \\
\cong \mid \theta \\
{\left[A, L_{0}\left(\Sigma B, X ;{ }^{*}\right)\right]} \\
\cong \mid \hat{\iota}_{*} \\
{\left[A, L_{0}(\Sigma B, X ; \iota)\right] \stackrel{i_{*}}{\longrightarrow}[A, L(\Sigma B, X ; \iota)] .}
\end{gathered}
$$

Proof. Let $f: A \# \Sigma B \rightarrow X$ represent an element of $[A \# \Sigma B, X]$. Then $\hat{\iota}_{*}^{\circ} \theta([f])$ has an associated map $F: A \times \Sigma B \rightarrow X$ given by

$$
F(a,(b, t))= \begin{cases}f(a,(b, 2 t) & 0 \leqq t \leqq \frac{1}{2} \\ \ell(b, 2 t-1) & \frac{1}{2} \leqq t \leqq 1\end{cases}
$$

Let $\phi: \Sigma(A \# \Sigma B) \rightarrow \Sigma(A \times \Sigma B)$ and $q: A \times \Sigma B \rightarrow A \# \Sigma B$ be as in the comments preceding Difinition 1.2. Consider the following diagram:

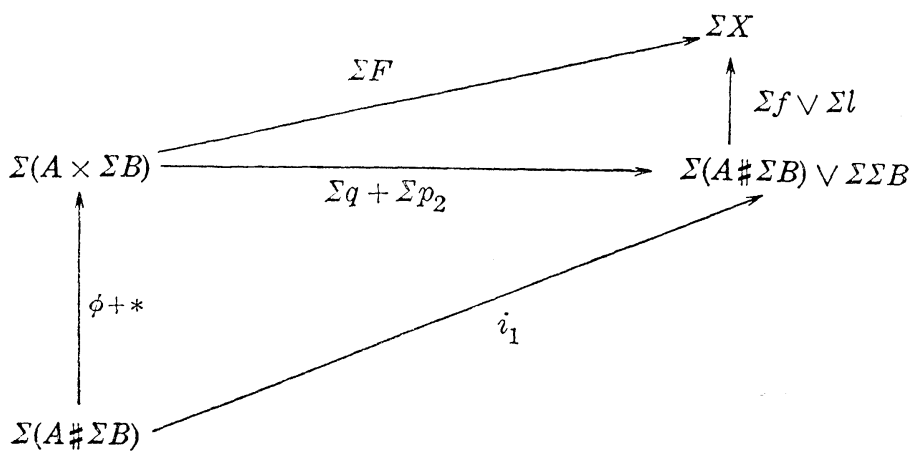

where $i_{1}$ is the inclusion of $\Sigma(A \# \Sigma B)$ in $\Sigma(A \# \Sigma B) \vee \Sigma \Sigma B$. The homotopy commutativity of this diagram will establish the result since $\Sigma F \circ(\phi+*) \cong \Sigma F \circ \phi$ which by definition is the element obtained from 
$\hat{\ell}_{*} \circ \theta([f])$ by the Hopf construction and $(\Sigma f \vee \Sigma \ell) \circ i_{1}=\Sigma f$, a representative of $E([f])$.

In the lower triangle of the diagram $\left(\Sigma q+\Sigma p_{2}\right) \circ(\phi+*) \cong \Sigma q \circ \phi$ which is homotopic to $i_{1}$ by the definition of $\phi$.

In the upper triangle

$$
\Sigma F(a,(b, t), r)= \begin{cases}(f(a,(b, 2 t)), r) & 0 \leqq t \leqq \frac{1}{2} \\ (\iota(b, 2 t-1), r) & \frac{1}{2} \leqq t \leqq 1\end{cases}
$$

and

$$
(\Sigma f \vee \Sigma \ell) \circ\left(\Sigma q+\Sigma p_{2}\right)(a,(b, t), r)= \begin{cases}(f(a,(b, t)), 2 r) & 0 \leqq r \leqq \frac{1}{2} \\ (\ell(b, t), 2 r-1) & \frac{1}{2} \leqq r \leqq 1\end{cases}
$$

The usual homotopy to interchange the roles of $t$ and $r$ for homotopy will work in this case since $f$ is defined on $A \# \Sigma B$ and $\ell$ is independent of $a$. Thus the upper triangle is homotopy commutative and the lemma is established.

DEFINITION 3.2. The classical EHP sequence is given by:

$$
\begin{gathered}
\pi_{3 n-2}\left(S^{n}\right) \stackrel{E}{\longrightarrow} \pi_{3 n-1}\left(S^{n+1}\right) \longrightarrow \cdots \longrightarrow \pi_{n+p}\left(S^{n}\right) \longrightarrow \\
\pi_{n+p+1}\left(S^{n+1}\right) \stackrel{H^{\prime}}{\longrightarrow} \pi_{p}\left(S^{n}\right) \stackrel{P}{\longrightarrow} \pi_{p+n-1}\left(S^{n}\right) \stackrel{E}{\longrightarrow} \cdots
\end{gathered}
$$

where $E$ is the suspension homomorphism, $H^{\prime}=-E^{-n-1} \circ \widetilde{H}$ where $\widetilde{H}$ is the Hopf invariant of Definition 1.8, $P=P_{\iota n}$, where $\iota_{n}=\left[1_{S^{n}}\right] \in \pi_{n}\left(S^{n}\right)$.

This sequence was shown exact in [7]; the form used in Definition 3.2 is that of P. J. Hilton and J. H. C. Whitehead in [3]. The classical $E H P$ sequence can now be compared with the $\iota_{n}$-component $E H P$ sequence for the fibration $\omega: L\left(S^{n}, S^{n} ; 1_{S^{n}}\right) \rightarrow S^{n}$.

THEOREM 3.3. For $q \leqq 3 n-2$ the following exact ladder is commutative and $H$ is an isomorphism:

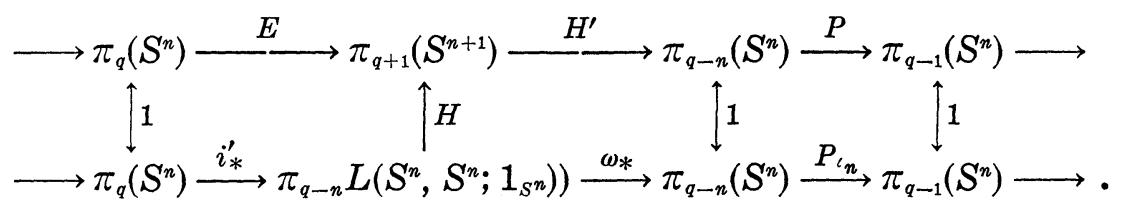

Proof. The left square commutes by Lemma 3.1 since, by defini- 
tion, $i_{*}^{\prime}=i_{*} \circ \hat{\ell}_{*} \circ \theta$. The right square commutes by the definition of $P$. For the range $q \leqq 3 n-2$, G. W. Whitehead shows (Corollary 6-4 in [7]) that every element $\alpha \in \pi_{q+1}\left(S^{n+1}\right)$ is obtainable from a map $F: S^{q-n} \times S^{n} \rightarrow S^{n}$ of type $\left(H^{\prime}(\alpha), \iota_{n}\right)$ by a Hopf construction. Thus if $F$ is considered as an associated map for an element $\beta \in$ $\pi_{q-n}\left(L\left(S^{n}, S^{n} ; 1_{S^{n}}\right)\right), \beta$ has type $\left(\omega_{*}(\beta), \iota_{n}\right)$ and $H(\beta)$ is obtainable by a Hopf construction from a map of type $\left(\omega_{*}(\beta), \iota_{n}\right)$ as well as a map of type $\left(H^{\prime}(\alpha), \iota_{n}\right)$. But then by 5.1 in [6], $\omega_{*}(\beta) * \iota_{n}=H^{\prime}(\alpha) * \iota_{n}$, where $*$ is the join operation. Since $\iota_{n}$ is the homotopy class of $1_{S^{n}}, E^{n+1} \omega_{*}(\beta)=$ $\omega_{*}(\beta) * \iota_{n}=H^{\prime}(\alpha) * \iota_{n}=E^{n+1} H^{\prime}(\alpha)$. Now $q \leqq 3 n-2$ so $q-n \leqq 2 n-2$ and by the Freudenthal suspension theorem $E^{n+1}$ is an isomorphism, thus $\omega^{*}(\beta)=H^{\prime}(\alpha)=H^{\prime}(H(\beta))$. This establishes the commutivity of the ladder. That $H$ is an isomorphism follows from the five lemma.

Since the bottom line is the $c_{n}$-component $E H P$ sequence, the classical $E H P$ sequence can be considered as the $\iota_{n}$-component $E H P$ sequence for spheres in the range $q \leqq 3 n-2$.

Some definitions will be required before describing the suspension sequence of James. Let $D^{n}$ denote the solid $n$-ball. Then $\partial D^{n}=S^{n-1}=$ $D_{+}^{n-1} \cup D_{-}^{n-1}$ where $D_{+}^{n-1}$ and $D_{-}^{n-1}$ are the northern and southern hemispheres of $S^{n-1}$ respectively. Note that $D_{+}^{n-1} \cap D_{-}^{n-1}=S^{n-2}$.

Definition 3.4. For $A$ and $B$ subspaces of $X$ such that $A \cap B \neq$ $\phi$ let $\pi_{n}(X ; A, B)$ be the set of homotopy classes maps of $f:\left(D^{n}, D_{+}^{n-1}\right.$, $\left.D_{-}^{n-1}\right) \rightarrow(X, A, B)$.

There are natural boundary operators $\partial_{1}: \pi_{n}(X ; A, B) \rightarrow$ $\pi_{n-1}(A, A \cap B)$ and $\partial_{2}: \pi_{n-1}(A, A \cap B) \rightarrow \pi_{n-2}(A \cap B)$ defined by restriction to $\left(D_{+}^{n-1}, S_{-}^{n-2}\right)$ and $S^{n-2}$ respectively.

DeFINITION 3.5. The repeated boundary operator $\Delta: \pi_{n}(X ; A, B) \rightarrow$ $\pi_{n-2}(A \cap B)$ is defined by $\Delta=\partial_{2} \circ \partial_{1}$.

The following result of James will be useful.

Theorem 3.6. There is a pairing $\{\beta, \gamma\} \in \pi_{p+q+1}\left(\Sigma X ; C_{+} X, C_{-} X\right)$ for $\beta \in \pi_{p}(X)$ and $\gamma \in \pi_{q}(X)$ such that

(i) $\Delta\{\beta, \gamma\}=[\beta, \gamma] \in \pi_{p+q+1}(X)$, the usual Whitehead product and

(ii) If $i_{*}: \pi_{p+q+1}(\Sigma X) \rightarrow \pi_{p+q+1}\left(\Sigma X ; C_{+} X, C_{-} X\right)$ is the natural inclusion, an element $\alpha \in \pi_{p+q+1}(\Sigma X)$ is obtainable by a Hopf construction of type $(\beta, \gamma)$ iff $i_{*}(\alpha)=\{\beta, \gamma\}$.

Proof. See $\S 4$ and Theorem 2.17 in [4].

DeFINITION 3.7. The James suspension sequence is 
$\longrightarrow \pi_{p+q}(X) \stackrel{E}{\longrightarrow} \pi_{p+q+1}(\Sigma X) \stackrel{i_{*}}{\longrightarrow} \pi_{p+q+1}\left(\Sigma X ; C_{+} X, C \_X\right) \stackrel{\Delta}{\longrightarrow} \pi_{p+q-1}(X)$

where $E$ is the suspension homomorphism and $\Delta$ is the repeated boundary operator.

THEOREM 3.8. The following exact ladder is commutative:

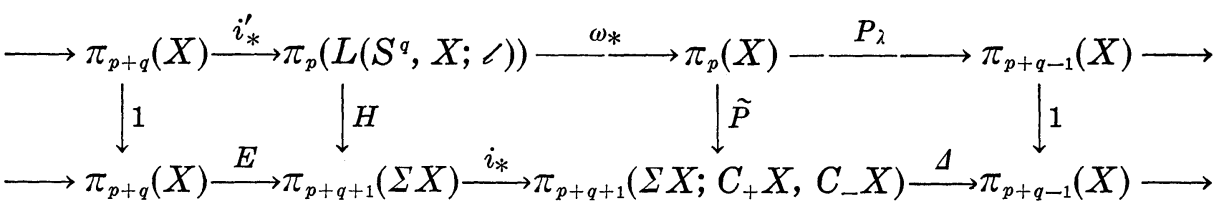

where $H$ is as in Lemma 3.1, $\widetilde{P(\alpha)}=\{\alpha, \lambda\}$, and $\lambda=[\ell]$ for any $[\ell] \in \pi_{q}(X)$.

Proof. The left square commutes by Lemma 3.1 since $i_{*}^{\prime}=i_{*}$ 。 $\hat{\ell}_{*} \circ \theta$ by definition. If $\alpha \in \pi_{p}\left(L\left(S^{q}, X ; \ell\right)\right)$ then by definition of $H, H(\alpha)$ is obtainable by a Hopf construction of type $\left(\omega_{*}(\alpha), \lambda\right)$ and by Theorem 3.6, (ii), $i_{*} H(\alpha)=\left\{\omega_{*}(\alpha), \lambda\right\}=\widetilde{P}_{\circ} \omega_{*}(\alpha)$. Thus the middle square commutes. The right square commutes by Theorem 3.6, (i).

Theorem 3.8 clearly indicates the extent to which the map $i_{*}^{\prime}=$ $i_{*} \circ \hat{\ell}_{*} \circ \theta$ of the $\lambda$-component $E H P$ sequence approximates the suspension homomorphism. Indeed, $E=H \circ i_{*}^{\prime}$. While the James sequence contains the suspension homomorphism in a straight forward form, the $\lambda$-component EHP sequence contains the generalized Whitehead product in a more direct form.

\section{REFERENCES}

1. Martin Arkowitz, The generalized Whitehead product, Pacific J. Math., 12 (1962), 7-23.

2. K. A. Hardie, A generalization of the Hopf construction, Quart. J. Math. Oxford Ser., (2) 12 (1961), 196-204.

3. Peter Hilton and J. H. C. Whitehead, Note on the Whitehead product, Ann. of Math.,

(2) 58 (1953), 429-442.

4. I. M. James, On the suspension triad, Ann. of Math., (2) 63 (1956), 191-247.

5. Edwin H. Spanier, Algebraic Topology, McGraw-Hill Book Company, New York 1966.

6. G. W. Whitehead, A generalization of the Hopf invariant, Ann. of Math., (2) 51 (1950), 192-237.

7. - On the Freudenthal theorems, Ann. of Math., (2) 57 (1953), 209-229.

Received June 7, 1971. This paper constituted part of the author's doctoral thesis written at Purdue University under Daniel H. Gottlieb.

FAIRFIELD UNIVERSITY 


\section{PACIFIC JOURNAL OF MATHEMATICS}

\section{EDITORS}

H. SAMELSON

Stanford University

Stanford, California 94305

C. R. Hовву

University of Washington

Seattle, Washington 98105
J. DugundJI

Department of Mathematics University of Southern California

Los Angeles, California 90007

RICHARD ARENS

University of California

Los Angeles, California 90024

\section{ASSOCIATE EDITORS}

E. F. BECKENBACH

B. H. NeumanN

F. WOLF

K. YoSHIDA

\section{SUPPORTING INSTITUTIONS}

UNIVERSITY OF BRITISH COLUMBIA

CALIFORNIA INSTITUTE OF TECHNOLOGY

UNIVERSITY OF CALIFORNIA

MONTANA STATE UNIVERSITY

UNIVERSITY OF NEVADA

NEW MEXICO STATE UNIVERSITY

OREGON STATE UNIVERSITY

UNIVERSITY OF OREGON

OSAKA UNIVERSITY
UNIVERSITY OF SOUTHERN CALIFORNIA

STANFORD UNIVERSITY

UNIVERSITY OF TOKYO

UNIVERSITY OF UTAH

WASHINGTON STATE UNIVERSITY

UNIVERSITY OF WASHINGTON

AMERICAN MATHEMATICAL SOCIETY

NAVAL WEAPONS CENTER

Printed in Japan by International Academic Printing Co., Ltd., Tokyo, Japan 


\section{Pacific Journal of Mathematics}

\section{Vol. 44, No. $1 \quad$ May, 1973}

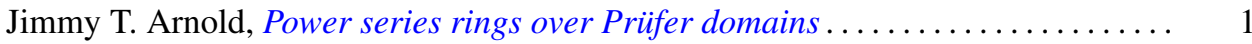

Maynard G. Arsove, On the behavior of Pincherle basis functions . . . . . . . . . 13

Jan William Auer, Fiber integration in smooth bundles ................. 33

George Bachman, Edward Beckenstein and Lawrence Narici, Function algebras

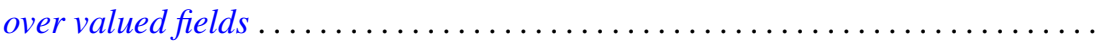

Gerald A. Beer, The index of convexity and the visibility function . . . . . . . . . . .

James Robert Boone, A note on mesocompact and sequentially mesocompact

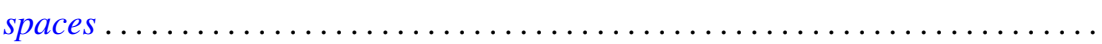

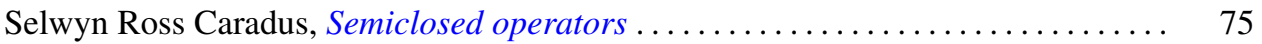

John H. E. Cohn, Two primary factor inequalities . . . . . . . . . . . . . . . 81

Mani Gagrat and Somashekhar Amrith Naimpally, Proximity approach to

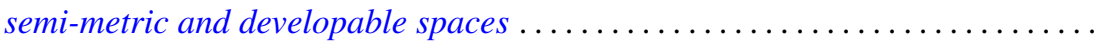

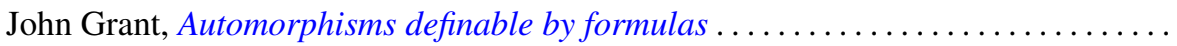

Walter Kurt Hayman, Differential inequalities and local valency ..............

Wolfgang H. Heil, Testing 3-manifolds for projective planes . . . . . . . . . . . . .

107

Melvin Hochster and Louis Jackson Ratliff, Jr., Five theorems on Macaulay

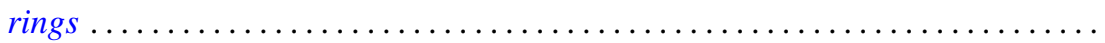

Thomas Benton Hoover, Operator algebras with reducing invariant subspaces ....

James Edgar Keesling, Topological groups whose underlying spaces are separable

Fréchet manifolds...

Frank Leroy Knowles, Idempotents in the boundary of a Lie group . .

191

George Edward Lang, The evaluation map and EHP sequences ...

201

Everette Lee May, Jr, Localizing the spectrum . . . . . . . . . . . .

211

Frank Belsley Miles, Existence of special $K$-sets in certain locally compact abelian groups.

Susan Montgomery, A generalization of a theorem of Jacobson. II . .

T. S. Motzkin and J. L. Walsh, Equilibrium of inverse-distance forces in

three-dimensions.

Arunava Mukherjea and Nicolas A. Tserpes, Invariant measures and the converse

of Haar's theorem on semitopological semigroups .

James Waring Noonan, On close-to-convex functions of order $\beta$

Donald Steven Passman, The Jacobian of a growth transformation

Dean Blackburn Priest, A mean Stieltjes type integral ........ .

Joe Bill Rhodes, Decomposition of semilattices with applications to topological

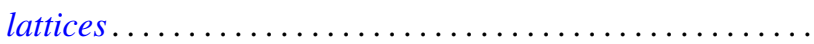

Claus M. Ringel, Socle conditions for $\mathrm{QF}-1$ rings ..........

Richard Rochberg, Linear maps of the disk algebra

Roy W. Ryden, Groups of arithmetic functions under Dirichlet convolution . .

Michael J. Sharpe, A class of operators on excessive functions

Erling Stormer, Automorphisms and equivalence in von Neumann algebras ..

Philip C. Tonne, Matrix representations for linear transformations on series

analytic in the unit disc. 